\title{
Effects of clonidine and scopolamine on multiple target detection in rapid serial visual presentation
}

\author{
Stephen B. R. E. Brown ${ }^{1,2}$ • Heleen A. Slagter ${ }^{3,5}$ - Martijn S. van Noorden ${ }^{4}$. \\ Erik J. Giltay ${ }^{4}$ Nic J. A. van der Wee ${ }^{2,4}$ - Sander Nieuwenhuis ${ }^{1,2}$
}

Received: 23 March 2015 / Accepted: 7 October 2015 / Published online: 28 October 2015

(C) The Author(s) 2015. This article is published with open access at Springerlink.com

\begin{abstract}
Rationale The specific role of neuromodulator systems in regulating rapid fluctuations of attention is still poorly understood.

Objectives In this study, we examined the effects of clonidine and scopolamine on multiple target detection in a rapid serial visual presentation task to assess the role of the central noradrenergic and cholinergic systems in temporal attention.

Method Eighteen healthy volunteers took part in a crossover double-dummy study in which they received clonidine (150/ $175 \mu \mathrm{g})$, scopolamine $(1.2 \mathrm{mg})$, and placebo by mouth in counterbalanced order. A dual-target attentional blink task was administered at $120 \mathrm{~min}$ after scopolamine intake and $180 \mathrm{~min}$ after clonidine intake. The electroencephalogram was measured during task performance.

Results Clonidine and scopolamine both impaired detection of the first target (T1). For clonidine, this impairment was accompanied by decreased amplitudes of the P2 and P3 components of the event-related potential. The drugs did not impair second-target (T2) detection, except if $\mathrm{T} 2$ was presented immediately after $\mathrm{T} 1$. The attentional blink for $\mathrm{T} 2$ was not
\end{abstract}

Stephen B. R. E. Brown

sbrebrown@gmail.com

1 Cognitive Psychology Unit, Institute of Psychology, Leiden University, Leiden, The Netherlands

2 Leiden Institute for Brain and Cognition, Leiden, The Netherlands

3 Brain and Cognition Unit, Department of Psychology, University of Amsterdam, Amsterdam, The Netherlands

4 Department of Psychiatry, Leiden University Medical Center, Leiden, The Netherlands

5 Amsterdam Brain and Cognition Center, University of Amsterdam, Amsterdam, The Netherlands affected, in line with a previous study that found no effect of clonidine on the attentional blink.

Conclusions These and other results suggest that clonidine and scopolamine may impair temporal attention through a decrease in tonic alertness and that this decrease in alertness can be temporarily compensated by a phasic alerting response to a salient stimulus. The comparable behavioral effects of clonidine and scopolamine are consistent with animal studies indicating close interactions between the noradrenergic and cholinergic neuromodulator systems.

Keywords Attentional blink $\cdot$ Rapid serial visual presentation $\cdot$ Temporal attention $\cdot$ Noradrenaline . Acetylcholine

\section{Introduction}

Temporal attention - the dynamic changes in attention on a fast timescale - is widely studied because it is crucial for organisms to be able to prioritize and accurately identify incoming information, for example, to make successful decisions. In recent years, the neuromodulatory basis of temporal attention has attracted considerable scientific interest. One theory relates temporal attention to the noradrenergic neuromodulator system. This theory is founded on the idea that the release of noradrenaline by the locus coeruleus (LC) adjusts the gain of post-synaptic neurons, thereby modulating these neurons' responsivity (Servan-Schreiber et al. (1990). The LC has been demonstrated to fire phasically following the presentation of task-relevant or salient stimuli (Aston-Jones et al. 2000), and these phasic bursts are temporally closely related to behavioral responses (Bouret and Sara 2004). These findings suggest that the phasic LC response acts as a temporal attention filter that selectively facilitates the processing of motivationally 
significantly stimuli (Aston-Jones and Cohen 2005; Nieuwenhuis et al. 2005a).

Rapid changes in temporal attention are commonly studied with the attentional blink task, in which participants have to identify two targets that are embedded in a rapid serial visual stream (RSVP) of distractor stimuli. Participants are usually able to accurately identify the first of those targets (T1). The crucial finding in this task is that when the second target (T2) follows the first target within 200-400 ms, participants are often unable to report T2 accurately (Raymond et al. 1992; Chun and Potter 1995). This phenomenon is referred to as the attentional blink.

Nieuwenhuis and colleagues have proposed a theory in which the attentional blink reflects the temporal dynamics of the noradrenergic system (Nieuwenhuis et al. 2005b; see also Warren et al. 2009). This theory assumes that identification of $\mathrm{T} 1$ is associated with a transient burst of arousal and concomitant phasic firing of the LC. Following this T1-related phasic burst, LC neurons enter a refractory period of reduced firing. During this period, no noradrenaline-mediated facilitation of stimulus processing can occur. The characteristic attentional blink window of 200-400 ms corresponds to the duration of this refractory period, which would explain why participants often fail to detect $\mathrm{T} 2$ if it is presented within this time window. This theory also accounts for the phenomenon of lag-1 sparing (Raymond et al. 1992; Hommel and Akyürek 2005): Participants generally do detect $\mathrm{T} 2$ if it directly follows $\mathrm{T} 1$ (i.e., at "lag 1"). When there is such a close temporal proximity of $\mathrm{T} 1$ and $\mathrm{T} 2$, detection of $\mathrm{T} 2$ is proposed to benefit from the phasic noradrenaline burst elicited by T1 (Usher et al. 1999).

There is direct empirical evidence for the involvement of the noradrenergic system in target detection under rapid serial visual presentation conditions. For example, antagonization of noradrenergic $\beta$ receptors by propranolol led to impaired $\mathrm{T} 2$ identification in humans (de Martino et al. 2008). Furthermore, patients with dopamine- $\beta$-hydroxylase deficiency, a rare genetic syndrome characterized by the complete absence of noradrenaline, were shown to have a larger attentional blink than healthy controls, and this impairment was restored by treatment with a synthetic precursor of noradrenaline (Jepma et al. 2011). However, when Nieuwenhuis et al. (2007) used the noradrenergic $\alpha_{2}$ agonist clonidine to attenuate noradrenergic baseline activity, they did not find a reliable decrease in T2 identification accuracy, a finding that seems at odds with the theory of Nieuwenhuis et al. (2005b).

The goal of the present experiment was to replicate the study by Nieuwenhuis et al. (2007) but with two improvements: a crossover design instead of a between-subject design to increase statistical power and an increased dose of clonidine to induce a more pronounced attenuation of the noradrenergic system. Furthermore, we recorded the electroencephalogram (EEG) to acquire more insight into the electrophysiological correlates of treatment effects on RSVP performance.
We tested 18 healthy adult participants in a double-blind placebo-controlled randomized crossover design. Participants received, in different test sessions, a single dose of clonidine, scopolamine, and placebo. Clonidine is a centrally acting $\alpha_{2}$ agonist that attenuates baseline noradrenergic activity by agonizing pre-synaptic $\alpha_{2}$ receptors and decreases the amplitude of the human P3 component, a putative electrophysiological correlate of phasic noradrenaline release (Nieuwenhuis et al. 2005a; Pineda et al. 1989). Due to its antihypertensive properties, the main indication of this drug is hypertension, but it is also indicated to reduce menopausal hot flashes and as an adjuvant in opiate withdrawal treatment. Its most common side effects are dizziness, sedation, orthostatic hypotension, and dry mouth. If the attentional blink is mediated by a phasic noradrenergic burst following presentation of $\mathrm{T} 1$ and clonidine decreases this phasic burst, then clonidine may be expected to reduce the attentional blink. Notably, previous eventrelated potential (ERP) studies have associated the attentional blink with a larger or a delayed T1-elicited P3 (Martens et al. 2006; Sergent et al. 2005; Slagter et al. 2007). We were thus specifically interested in possible effects of clonidine on the T1-elicited P3.

The involvement of the cholinergic system in temporal attention has been investigated less extensively than that of noradrenaline. Previous empirical work has focused on the nicotinic cholinergic system and has not studied RSVP performance but other temporal attention tasks like temporal cuing (e.g., Beane and Marrocco 2004; Stewart et al. 2001). Therefore, we also administered the muscarinic antagonist scopolamine, a drug with a sedation profile comparable to that of clonidine. Scopolamine has pronounced antiemetic properties, and its main indications are therefore (post-operative) nausea and motion sickness, but it is also indicated to treat gastrointestinal spasms. Its main side effects are dry mouth, bradycardia, and mydriasis. The use of scopolamine allowed us to gain insight into the role of the cholinergic muscarinic system in temporal attention and to test whether any treatment effects are specific to the noradrenergic system.

\section{Methods and materials}

\section{Participants}

Eighteen healthy young adults (15 women), aged 18-26 years (mean age 21 years), drafted through Leiden University's participant recruitment system, took part in three 4.5-h experimental sessions in return for $€ 140$. Only participants with a systolic blood pressure above $100 \mathrm{mmHg}$, a diastolic blood pressure above $70 \mathrm{mmHg}$, and a heart frequency over 65 beats per minute in rest were included in the study (cf. Nieuwenhuis et al. 2007). All participants underwent a medical screening which included a routine physical examination prior to being 
included in the experiment: Only healthy individuals without a history of neurological or psychiatric disorders were allowed to participate. Participants took no prescribed medication and did not smoke; participants were instructed to abstain from using recreational drugs, caffeine, or alcohol $15 \mathrm{~h}$ prior to the study. Female participants were asked whether they were pregnant or thought they might be pregnant to preclude pregnant females from participating. Participants received a single oral dose of clonidine, a single oral dose of scopolamine $(1.2 \mathrm{mg})$, and a placebo in a randomized, double-blind, counterbalanced double-dummy crossover design. The first 11 participants received a clonidine dose of $175 \mu \mathrm{g}$. As the 11th participant showed an unexpected large drop in blood pressure of $35 \mathrm{mmHg}$ systolic, but without clinical consequences, 60 min after the ingestion of clonidine $175 \mu \mathrm{g}$ (blind was broken by the supervising physician), we decided to reduce the dose of clonidine to $150 \mu \mathrm{g}$ for the final seven participants. Preliminary repeated measures ANOVAs with dose as between-subject factor revealed no reliable main effect of dose or interactions including this factor, so in the analyses reported below, the 18 participants are pooled. Clonidine, scopolamine, and placebo were administered during three separate test sessions, spaced 1 week apart. The study was approved by the medical ethics committee of the Leiden University Medical Center. Written informed consent was obtained from all participants prior to inclusion in the study.

\section{Task}

Participants performed an attentional blink task. Each trial started with a 500-ms fixation point (black plus sign on light gray background, visual angle $0.6^{\circ} \times 0.6^{\circ}$ ), followed by a 2 -s blank, after which a RSVP stream of 22 uppercase letters was presented centrally (visual angle of each letter approximately $0.7^{\circ} \times 0.7^{\circ}$ ) on an IIyama Vision Master CRT monitor with a refresh rate of $100 \mathrm{~Hz}$, using E-Prime 2.0 (Psychology Software Tools, Sharpsburg, PA). Each letter was randomly drawn without replacement from the alphabet and presented for $70 \mathrm{~ms}$, followed by a blank of $30 \mathrm{~ms}$. The letters $I, O, Q$, and $S$ were left out, as they resemble digits too much. On each trial, two letters were replaced by digits (range $2-9$, chosen randomly without replacement): targets 1 and 2 (T1 and T2). $\mathrm{T} 2$ was presented three to six temporal positions from the end of the stream. The temporal distance between $\mathrm{T} 1$ and $\mathrm{T} 2$ was either one (12.5\% of trials), two (37.5\% of trials), three ( $37.5 \%$ of trials), or seven items ( $12.5 \%$ of trials), corresponding to lags of 100, 200, 300, and $700 \mathrm{~ms}$. Immediately after the end of the RSVP stream, participants were asked to identify $\mathrm{T} 1$ and $\mathrm{T} 2$ by typing them, in order, on a standard keyboard. The task consisted of six blocks of 40 trials each and was preceded by a practice block of 12 trials, in which feedback on the participants' performance was given on every trial (e.g., a display of "+ -" indicated that a participant had entered T1 correctly and T2 incorrectly).

\section{Procedure}

Each participant was tested at approximately the same time of day. During every test session, participants received a capsule of clonidine or placebo at 09.35 AM and a capsule of scopolamine or placebo at 10.35 AM. The different kinetic profiles of clonidine and scopolamine necessitated administrations at different times prior to testing. This double-dummy design resulted in one clonidine session (i.e., clonidine verum plus scopolamine placebo), one scopolamine session (clonidine placebo plus scopolamine verum), and one placebo session (clonidine plus scopolamine placebos). To eliminate any possible confound of drug order, we stratified this factor by distributing the six possible drug orders evenly across participants.

The procedure in each test session is illustrated in Fig. 1. At the start of each session ( $t=-20 \mathrm{~min}$ ), a peripheral intravenous cannula was placed and connected to an intravenous $0.9 \%$ $\mathrm{NaCl}$ (saline) drip to be able to increase blood pressure through volume expansion and to have an entryway to administer escape medication in the case of a severe drop in tension and/or heart frequency. Furthermore, three cardio electrodes were applied to the participant's chest and connected to an electrocardiography (ECG) monitor. To measure the sedative, alertness-reducing properties of clonidine and scopolamine,
Fig. 1 Timeline of the procedure in each test session. Connector lines indicate the start of an event. Each cognitive task lasted approximately $30 \mathrm{~min}$. Participants' blood pressure and heart rate were measured at baseline $(t=-20)$ and then every $15 \mathrm{~min}$, starting at $t=0$. Results from the other cognitive tasks are reported elsewhere (asterisk) (Brown et al. 2015a, b). SRT simple reaction time task

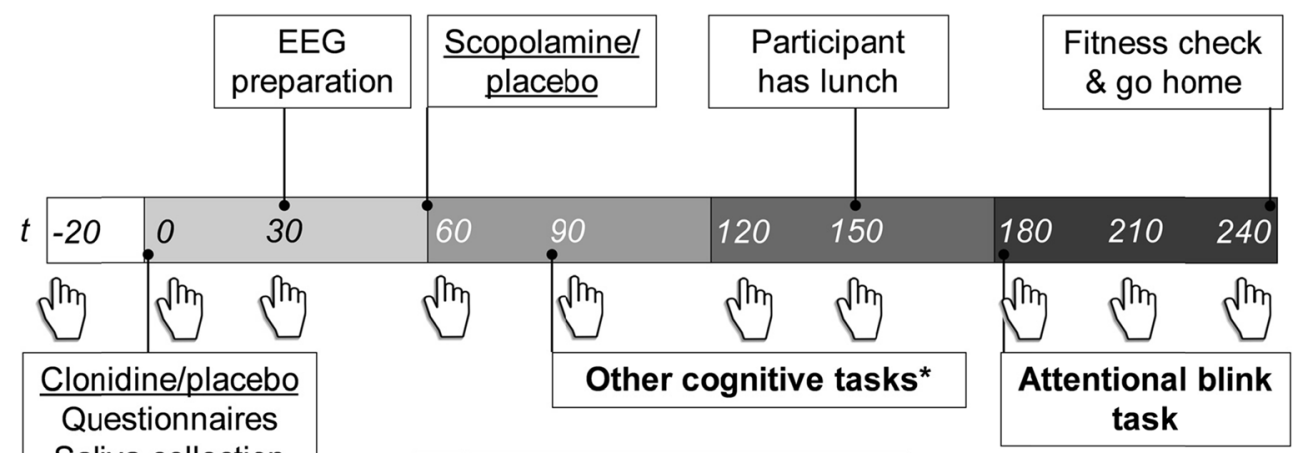

Participant completes SRT 
we administered a 40-trial simple reaction time task upon a participant's arrival in the lab, as well as right before and after the participant performed the attentional blink task. Participants had to respond as quickly as possible whenever a white circle appeared on the computer screen. Stimulus onset asynchrony was jittered between 500 and $1250 \mathrm{~ms}$, with a mean of $1000 \mathrm{~ms}$; this task lasted less than $2 \mathrm{~min}$.

At $t=0 \mathrm{~min}$, participants ingested a microcrystalline cellulose-filled capsule with either clonidine or placebo. Clonidine has well-established antihypertensive properties: Therefore, blood pressure and heart rate were monitored four times an hour from $t=0$ onwards for participant safety with an Omron M10-IT automatic sphygmomanometer. At $t=60 \mathrm{~min}$, participants ingested a microcrystalline cellulose-filled capsule with either scopolamine or placebo.

At $t=180$, participants performed the attentional blink task which lasted approximately $30 \mathrm{~min}$; during the $90 \mathrm{~min}$ prior to this time point, participants performed three unrelated cognitive tasks (Brown et al. 2015a, b). Participant fitness was checked at $t=240$, and participants were sent home via public transportation if their blood pressure and heart rate were close to the values measured at $t=-20$; if their blood pressure and heart rate had not returned to normal yet, they were kept for further observation. At the end of the third test session, participants received their financial compensation.

\section{EEG recording and analyses}

We recorded EEG from $64 \mathrm{Ag} / \mathrm{AgCl}$ scalp electrodes and from the left and right mastoids. We measured the horizontal and vertical electro-oculogram (EOG) using bipolar recordings from electrodes placed approximately $1 \mathrm{~cm}$ lateral of the outer canthi of the two eyes and from electrodes placed approximately $1 \mathrm{~cm}$ above and below the participant's right eye. The EEG signal was pre-amplified at the electrode to improve the signal-to-noise ratio and amplified with a gain of $16 \times$ by a BioSemi ActiveTwo system (BioSemi B.V., Amsterdam). The data were digitized at 24-bit resolution with a sampling rate of $512 \mathrm{~Hz}$ using a low-pass fifth-order sinc filter with a half-power cutoff of $102.4 \mathrm{~Hz}$. Each active electrode was measured online with respect to a common mode sense (CMS) active electrode producing a monopolar (nondifferential) channel and was referenced offline to the average of the left and right mastoids. Data were high-pass filtered at $0.1 \mathrm{~Hz}$ and low-pass filtered at $30 \mathrm{~Hz}$. Ocular and eyeblink artifacts were corrected using the method of Gratton et al. (1983). Epochs with other artifacts (a gradient greater than $30 \mu \mathrm{V}$, slow drifts [>300 $\mu \mathrm{V} / 200 \mathrm{~ms}]$, and low activity $[<0.50 \mu \mathrm{V} / 100 \mathrm{~ms}]$ ) were discarded (placebo $1.2 \%$, clonidine $1.3 \%$, and scopolamine $2.5 \%$ ). Data were epoched from -100 to $600 \mathrm{~ms}$ relative to the onset of T1 and then averaged. A baseline, computed as the average signal activity across the 100 ms prior to T1, was subtracted for each ERP. Data pre- processing was performed in Brain Vision Analyzer 2 (Brain Products, Gilching, Germany).

As the active compounds only reliably influenced $\mathrm{T} 1 \mathrm{ac}-$ curacy (regardless of lag) and given previous studies linking the T1-evoked P3 to the attentional blink (e.g., Martens et al. 2006; Sergent et al. 2005; Slagter et al. 2007), we focused our electrophysiological analyses on T1-evoked potentials. Using MATLAB (The MathWorks, Natick, MA), we analyzed the ERP elicited by $\mathrm{T} 1$ with a sliding-window approach to examine whether clonidine and scopolamine differed from placebo, focusing in particular on electrodes $\mathrm{Cz}, \mathrm{CPz}$, and $\mathrm{Pz}$, where the P3 was largest in amplitude. We collapsed T1-locked ERPs across lags, split the ERPs for each treatment and each participant into 19.5 -ms windows, starting at $t=0$ (i.e., $0-19.5$, 21.5-39 ms, etc.), and then, for each window separately, submitted the average amplitudes to paired sample $t$ tests with treatment (clonidine or scopolamine vs. placebo) as independent variable.

\section{Results}

Greenhouse-Geisser corrections were applied whenever the assumption of sphericity was violated; in such cases, uncorrected degrees of freedom are reported.

\section{Physiological and alertness data}

Figure 2a shows that, as expected, clonidine lowered systolic (mean tension $101 \mathrm{mmHg}$ ) and diastolic $(65 \mathrm{mmHg})$ blood pressure relative to placebo (mean tension $112 / 73 \mathrm{mmHg}$ ), also during performance of the attentional blink task $(t=$ $180-210)$, all $t_{17}>4.5, p s<.0005$. The difference in systolic and diastolic blood pressure between placebo and scopolamine was not significant. Figure $2 \mathrm{~b}$ shows that scopolamine (61/min), as expected, lowered heart frequency relative to placebo $(71 / \mathrm{min})$ and clonidine $(69 / \mathrm{min})$, also during performance of the attentional blink task, all $t_{17}>3.3, p s<.004$.

To test the subjects' alertness, we administered a simple reaction time task at baseline (arrival of participant), right before, and right after performing the attentional blink task. As expected, the baseline measurements did not differ between treatments, $F(2,34)<1, p=.95$. To examine the effect of treatment on pre- and post-test measurements (Fig. 2c), we subtracted the baseline values from each of these measurements and submitted the difference scores to a 3 (treatment) $\times 2$ (time point) repeated measures ANOVA. This analysis yielded a main effect of treatment, $F(2,34)=4.8, p=.019$, partial $\eta^{2}=.22$, but no interaction between treatment and time point, $F(2,34)=1.6, p=.22$. Pairwise comparisons indicated that clonidine and scopolamine reliably slowed down simple reaction time compared to placebo during both the pre-test ( $t_{17}=2.1, p=.05$ and $t_{17}=2.3, p=.04$, respectively) and the 
Fig. 2 a Blood pressure data for the three treatments. The shaded gray area indicates significant pairwise comparisons between clonidine and placebo $(p<.05)$. b Heart frequency for the three treatments. The shaded gray area indicates significant pairwise comparisons between scopolamine and placebo $(p<.05)$. c Results from a simple reaction time task, administered at the start of the test session (baseline) and right before (pretest) and after (post-test) participants performed the attentional blink task
A

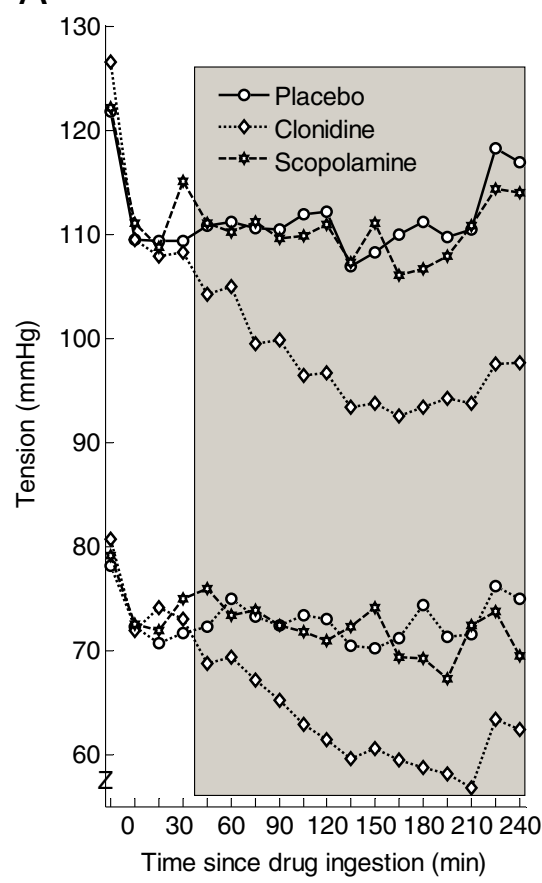

B

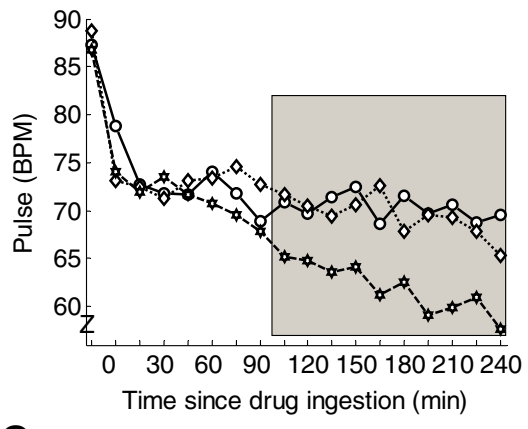

C

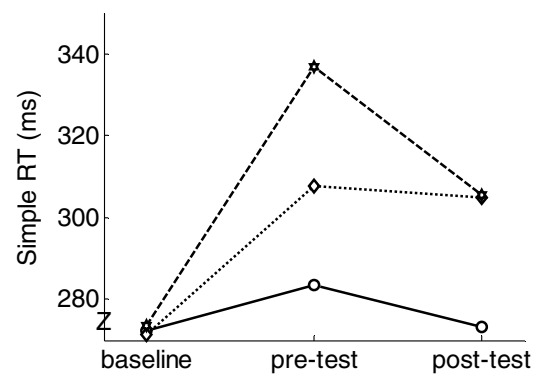

post-test $\left(t_{17}=3.1, p=.006\right.$ and $t_{17}=3.3, p=.005$, respectively). The differences between clonidine and scopolamine were not significant.

\section{Behavioral data}

Trials on which T1 and T2 were accurately identified but in the wrong order were treated as correct (cf. Nieuwenhuis et al. 2007). Thus, the probability of guessing T1 and T2 correctly was $3.6 \%$.

Figure 3 (left panel) shows average T1 accuracy as a function of treatment and lag. The main effect of treatment was significant, $F(2,34)=4.4, p=.02$, partial $\eta^{2}=.21$. Both clonidine $\left(79.3 \%, t_{17}=2.5, p=.02\right)$ and scopolamine $\left(79.4 \%, t_{17}=\right.$ 2.4, $p=.03)$ decreased T1 identification accuracy relative to placebo $(85.6 \%)$. T1 identification accuracy increased with lag, $F(3,51)=6.6, p=.001$, partial $\eta^{2}=.28$. Treatment and lag did not interact, $F(6,102)=0.2, p=.96$.

To determine if the two drugs also decreased T2 accuracy, we examined average T2 accuracy, non-contingent on T1 identification, as a function of treatment and lag (Fig. 3, middle panel). Treatment did not reliably influence $\mathrm{T} 2$ identification accuracy, $F(2,34)=1.6, p=.21$, partial $\eta^{2}=.09$. T2 identification performance showed the characteristic pattern of lag-1 sparing, a subsequent decrease of accuracy for lags 2 and 3 (i.e., the attentional blink), and a recovery of performance for lag $7, F(3,51)=12.1, p<.0005$, partial $\eta^{2}=.42$. Treatment and lag did not interact $(p=.051)$. We found it remarkable that the treatment effects for lag 1 were of a similar magnitude as those on T1 accuracy. Indeed, although there was no overall effect of treatment, analysis of individual lags yielded a reliable effect of treatment for lag $1, F(2,34)=5.8$, $p=.007$, partial $\eta^{2}=.25$, but not for the other three lags (all $p \mathrm{~s}>$.44). Pairwise comparisons for lag 1 revealed that accuracy in the clonidine $\left(76.6 \% ; t_{17}=3.1, p=.007\right)$ and scopolamine $\left(79.1 \% ; t_{17}=2.9, p=.009\right)$ conditions was lower than in the placebo condition $(87.7 \%)$, indicating that the treatment effects for T1 extended into lag 1 but not further. There was no reliable difference in accuracy between the clonidine and scopolamine conditions $(p=.51)$.

Figure 3 (right panel) shows the results of a similar T2 analysis but constrained to T1-correct trials, as is common in attentional blink research. This analysis yielded similar statistical results. Treatment did not reliably influence T2 identification accuracy, $F<1$. There was a main effect of lag, $F(3$, $51)=18.6, p<.0005$, partial $\eta^{2}=.52$, but no interaction between treatment and lag $(p=.33)$. Again, separate analyses of the four lags yielded a reliable effect of treatment for lag 1 only, $F(2,34)=3.3, p=.05$, partial $\eta^{2}=.16$. Pairwise comparisons for lag 1 revealed that T2 accuracy was lower in the clonidine $\left(92.3 \%, t_{17}=2.3, p=.04\right)$ and scopolamine $\left(t_{17}=\right.$ $2.1, p=.05)$ conditions than in the placebo condition $(97.5 \%)$. There was no reliable difference in accuracy between the clonidine and scopolamine conditions $(p=.40)$.

\section{Electrophysiological data}

Because clonidine and scopolamine only reliably affected $\mathrm{T} 1$ accuracy, we next examined the effects of drugs on T1-evoked ERPs, separately for T1-correct trials (Fig. 4a) and for all 
Fig. 3 T1 identification accuracy (left panel), $\mathrm{T} 2$ identification accuracy (middle panel), and T2 identification accuracy (conditional upon $\mathrm{T} 1$ correct) as a function of treatment and lag

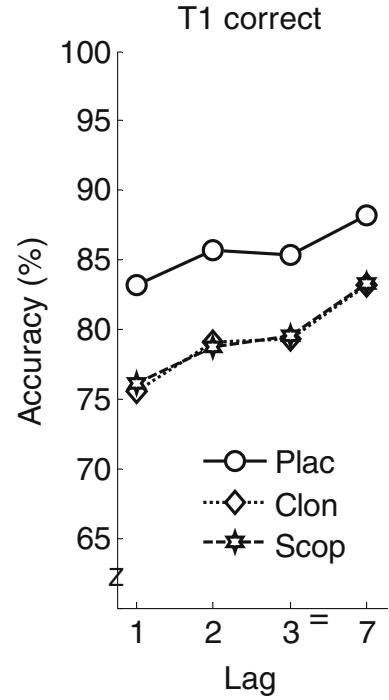

T2|T1 correct

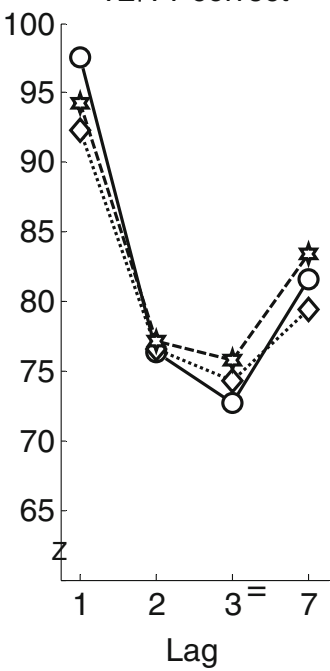

trials, regardless of $\mathrm{T} 1$ accuracy (Fig. 4b). Importantly, as is evident in Fig. 4, the drug-related waveforms lagged behind the placebo-related waveform, which poses a problem for our sliding-window approach, because amplitude differences may in fact reflect latency differences. To deal with this issue, we first determined the size of the lag for each drug. To do so, we performed cross-correlations between the grand average waveforms for placebo and clonidine and for placebo and scopolamine (cf. Śmigasiewicz et al. 2014). We then shifted, sample by sample (in $\sim 2$-ms steps), the drug-related waveform relative to the placebo-related waveform and computed the cross-correlation across the time interval $[0,500 \mathrm{~ms}]$. The maximum correlations were obtained by shifting the clonidine waveform 4 samples ( $\sim 8 \mathrm{~ms}$ ) to the left and by shifting the scopolamine waveform 2 samples ( $\sim 4 \mathrm{~ms}$ ) to the left. To correct for these lags, we next shifted the clonidine- and scopolamine-related waveforms of each subject by the corresponding duration. Grand average latency-corrected waveforms are shown in Fig. 4c, d. All ERP analyses reported below were based on these latency-corrected waveforms.

First, we examined the ERP waveforms for T1-correct trials. As can be seen in Fig. 4c, the sliding-window approach led to the identification of one significant time interval for electrode Cz: $217-273 \mathrm{~ms}$. In this P2 window, clonidine was associated with a smaller mean amplitude $(2.1 \mu \mathrm{V})$ than placebo $(3.0 \mu \mathrm{V}), t_{17}=2.9, p=.01$. Scopolamine $(2.5 \mu \mathrm{V})$ did not differ from placebo, $t_{17}=1.1, p=.28$.

Second, we examined the ERP waveforms based on all trials (i.e., regardless of $\mathrm{T} 1$ accuracy). The motivation for this analysis was that we were interested in the neural signatures associated with the drug-related impairment in T1 performance. Because several subjects made too few T1 errors to compute reliable error-specific ERP waveforms, we examined ERP waveforms based on all trials as neural signatures most
Fig. 4 T1-locked grand average ERP waveforms for electrode $\mathrm{Cz}$, plotted separately for each treatment. The horizontal black bars indicate time intervals where clonidine differed from placebo (see Methods and materials). Upper panels: original waveforms; lower panels: latency-corrected waveforms (see Results)
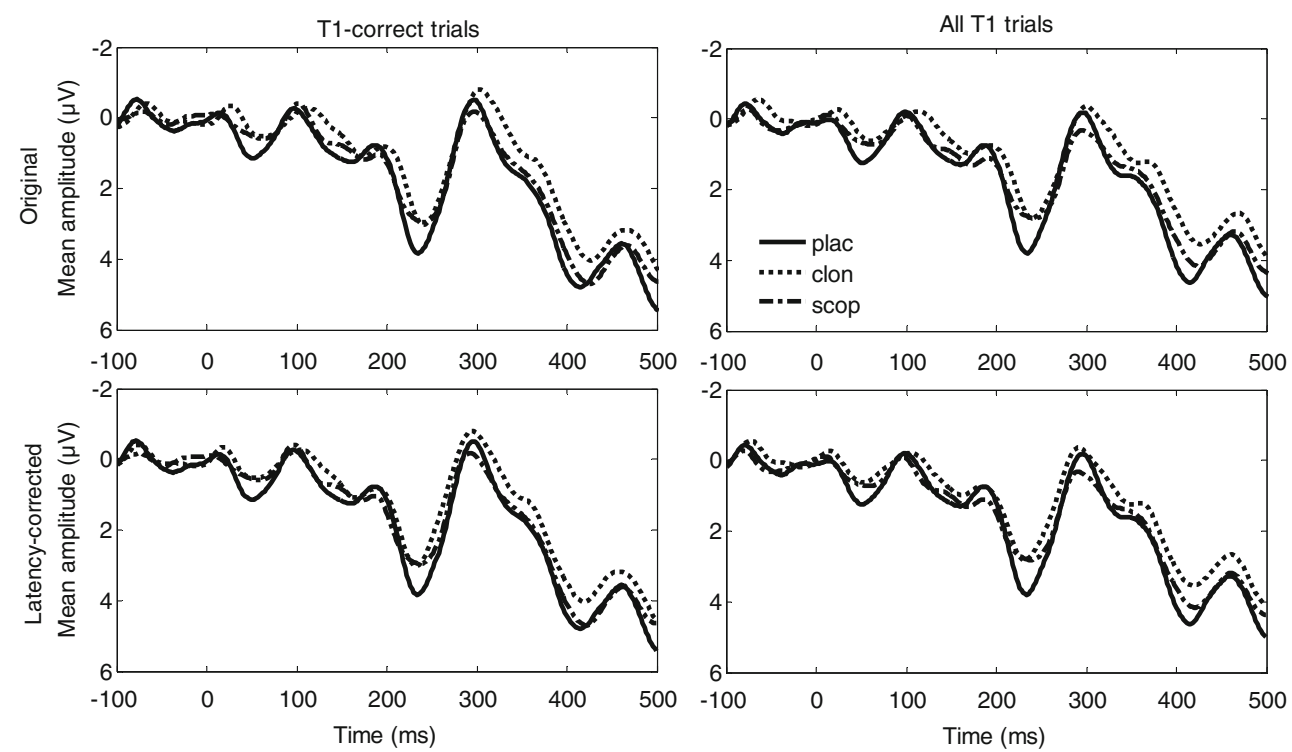
representative of overall $\mathrm{T} 1$ performance. Here, the slidingwindow approach led to the identification of two significant time intervals for electrode $\mathrm{Cz}$ (Fig. 4d): 217-273 ms, corresponding to the $\mathrm{P} 2$ component, and $373-430 \mathrm{~ms}$, corresponding to the P3. In the P2 interval, clonidine was again associated with a smaller mean amplitude $(2.1 \mu \mathrm{V})$ than placebo $(2.9 \mu \mathrm{V}), t_{17}=3.0, p=.009$, replicating the analysis based on $\mathrm{T} 1$-correct trials. In the $\mathrm{P} 3$ interval, clonidine was also associated with a smaller mean amplitude $(2.9 \mu \mathrm{V})$ than placebo $(3.8 \mu \mathrm{V}), t_{17}=2.9, p=.01$. Both the P2 effect and the P3 effect were also significant for electrode $\mathrm{CPz}$, suggesting a centroparietal locus of these effects. Examination of lagspecific T1-evoked ERPs indicated that the P2 and P3 effects were also present for longer lags, excluding the possibility that these effects constituted treatment effects on T2-related potentials that confounded the T1-evoked waveforms. Amplitudes for scopolamine generally lay in between clonidine and placebo (P2 interval $2.4 \mu \mathrm{V}$, P3 interval $3.5 \mu \mathrm{V}$ ) but did not differ reliably from placebo, both $p \mathrm{~s}>.15$.

Thus, relative to placebo, clonidine attenuated the amplitudes of the P2 and P3 components evoked by T1, although the latter effect was only reliable in the ERP analysis including correct and incorrect trials. Given that missed targets are typically associated with decreased P3 amplitudes (e.g., Rolke et al. 2001; dell' Acqua et al. 2003), the effect of clonidine on $\mathrm{P} 3$ amplitude must have been driven at least in part by the increased number of included T1-incorrect trials.

\section{Discussion}

In the present research, we investigated the effects of clonidine and scopolamine on multiple target detection in an RSVP context. In line with Nieuwenhuis et al. (2007), we found no effect of treatment on the attentional blink for T2. In contrast, we found that both clonidine and scopolamine impaired T1 accuracy. For clonidine, this effect was accompanied by a significant reduction in T1-evoked P2 and P3 amplitude.

The current study replicated Nieuwenhuis et al. (2007) with two design improvements: a crossover design instead of a between-subject design and a higher dose of clonidine, although, after a clinically relevant drop in blood pressure in one participant, the final seven participants were given the clonidine dose that was used by Nieuwenhuis et al. (2007). Like Nieuwenhuis et al. (2007), we found no effect of clonidine on the attentional blink, which poses a challenge for the attentional blink theory of Nieuwenhuis et al. (2005b) under the assumption that clonidine affects the phasic LC response. However, the evidence that addresses this assumption is limited. As in the present study, clonidine has been found to attenuate the amplitude of the P3 (Joseph and Sitaram 1989), which has been proposed to reflect phasic LC activity (Nieuwenhuis et al. 2005a). Furthermore, the suppressive effect of clonidine on tonic LC activity (e.g., Abercrombie and Jacobs 1987; Adams and Foote 1988), along with the reported interaction between tonic and phasic activity of the LC (Aston-Jones and Cohen 2005), suggests that clonidine may also affect sensory-evoked LC responses. However, the only study that we are aware of that directly examined this issue found mixed results: Adams and Foote (1988) found that during the onset of clonidine-induced suppressed LC firing, LC responses to sensory (footshock) stimuli were relatively preserved, although later during the experiment, the reliability of sensory-evoked LC responses was greatly reduced. Furthermore, in another experiment conducted as part of this study, we failed to find evidence that clonidine modulated the phasic alerting response to a task-irrelevant, auditory ("accessory") stimulus, despite it having an effect on general alertness (Brown et al. 2015a). Thus, we may not have found an effect of clonidine on the attentional blink because it is possible that phasic LC responses to RSVP targets were preserved.

Alternatively, if clonidine affects the phasic LC response, as the observed reduction in T1-evoked P3 amplitude might indicate, the theory of Nieuwenhuis et al. (2005b) could be incorrect. For example, the size and the duration of the LC refractory period, which is purportedly mirrored in the attentional blink, may not be proportional to the size of the phasic LC response, as the theory suggests, or noradrenaline may not be involved in the attentional blink at all. At first sight, the study by de Martino et al. (2008) suggests a role of the noradrenergic system in the attentional blink: These authors found that administration of the $\beta$-adrenoceptor antagonist propranolol decreased $\mathrm{T} 2$ accuracy, while administration of the noradrenergic reuptake inhibitor reboxetine increased accuracy for emotional T2 stimuli. However, the authors found no interactions between lag and treatment, and in one of their experiments, propranolol impaired T1 accuracy as well. Taken together, these findings suggest that propranolol and reboxetine do not specifically modulate the attentional blink but target detection under RSVP conditions in general. More convincing evidence for noradrenergic modulation of the attentional blink was provided by Jepma et al. (2011), who studied patients with dopamine- $\beta$-hydroxylase deficiency, a rare genetic syndrome characterized by the complete lack of noradrenaline. They found that these patients had a larger attentional blink than healthy controls and that this impairment was restored by treatment with a synthetic precursor for noradrenaline. Although these findings pose a challenge for the theory of Nieuwenhuis et al. (2005a), which explains the attentional blink as a by-product of phasic noradrenaline release in the LC, they are generally consistent with a role for noradrenaline in the attentional blink. Recent studies have also reported evidence that decreased levels of dopamine in the striatum are associated with a larger attentional blink (Colzato et al. 2011; Colzato et al. 2008; Slagter et al. 2012). 
Other neurotransmitters may hence also play a role in the attentional blink.

In our study, clonidine had a clear detrimental effect on T1 identification accuracy. Presumably, clonidine impaired performance by reducing general alertness (e.g., Brown et al. 2015a; Coull et al. 1997; Coull 2001; Smith and Nutt 1996), a possibility that is supported by the negative effects of clonidine on simple reaction time, and the fact that scopolamine, which also increased simple reaction time, similarly reduced T1 identification accuracy. Nieuwenhuis et al. (2007) did not find a significant effect of clonidine on T1 accuracy. However, in their study, all participants took the lower clonidine dose, and their effect was in the same direction and of a similar magnitude $(5 \%)$ as the effect we observed here $(6 \%)$. To further understand the effect of clonidine on T1 accuracy, we examined T1-related ERP waveforms (Kenemans and Kähkönen 2011).

Clonidine attenuated the amplitude of the T1-evoked P2 and P3 components. The functional significance of the P2 is relatively ill-defined, but it has been related to some aspect of stimulus classification (reviewed in Key et al. 2005). The opposite seems to apply for the P3: Since its discovery in 1965, a number of theories have been proposed to account for its functional significance. In the context of the current paper, the work by Nieuwenhuis et al. (2005a) is particularly relevant, as these authors conceptualized the P3 as reflecting phasic noradrenergic activity and the concomitant increase in neural gain. As noted above, the clonidine-related decrease in P3 amplitude is consistent with several previous studies (Nieuwenhuis et al. 2005a; Joseph and Sitaram 1989). In contrast, previous studies have reported no effect of clonidine on the amplitude of the P2 (Abuljawad et al. 2001; Turetsky and Fein 2002). We propose that the effects of clonidine on T1evoked P2 and P3 amplitudes and corresponding behaviors were mediated by a general decrease in alertness.

If clonidine reduced general alertness, why is that not manifested in reduced overall T2 accuracy? Relatedly, why did clonidine reduce lag-1 sparing? We propose that the perception of $\mathrm{T} 1$ caused a phasic alerting response that temporarily compensated for the drug-induced decrease in tonic alertness. As we have shown in other work, drug-related reductions in alertness yield room for compensatory accessory stimulusinduced performance improvements (Brown et al. 2015a). In a similar vein, Smith and Nutt (1996) found that arousal evoked by white noise can reduce the frequency of attentional lapses induced by clonidine intake. Furthermore, we propose that this phasic alerting response takes some time to unfold. This is suggested by our finding that the drug-related impairments in T1 accuracy extended to T2 accuracy if T2 was presented immediately after T1 (i.e., at lag 1). Only after that, from lag 2 onward, did accuracy return to placebo levels.

The scopolamine findings show a remarkable similarity to the clonidine findings. Like clonidine, scopolamine reduced
T1 accuracy without having a clear effect on T2 accuracy. The reduction in $\mathrm{T} 1$ accuracy is generally consistent with a number of studies that have reported scopolamine-induced attentional impairments, as indicated by impaired performance in sustained attention tasks (Hasselmo and Sarter 2011). Scopolamine also led to reduced amplitudes of the P2 and $\mathrm{P} 3$ relative to placebo, although these reductions were not statistically significant.

It is possible that the effects of clonidine and scopolamine on behavior and ERP waveforms, though similar, were achieved via largely independent neural pathways that both affect general alertness. However, we believe that it is more plausible that the similar effects of these two drugs in the current study and another recent study in our lab (Brown et al. 2015a) reflect interactions between the two neuromodulator systems involved (Briand et al. 2007). On one hand, acetylcholine has been demonstrated to activate LC neurons in rats and co-administration of scopolamine reduces this effect (Egan and North 1985; Adams and Foote 1988). Egan and North proposed that scopolamine antagonizes muscarinic receptors in the LC, leading to reduced noradrenergic baseline activation. On that assumption, both clonidine and scopolamine may have reduced noradrenergic baseline activity, leading to a similar pattern of results for both treatments. On the other hand, there is solid evidence that clonidine inhibits cortical ACh release (Acquas et al. 1998), probably via $\alpha_{2}$ receptors in the basal forebrain (cf. Dringenberg and Vanderwolf 1998). This suggests that both clonidine and scopolamine may have reduced basal forebrain activity and consequent release of acetylcholine, thus leading to a similar pattern of results. The current data underline the importance of studying interactions between the noradrenergic and cholinergic neuromodulator systems in regulating temporal fluctuations in attention.

Some limitations of this study are worth mentioning. First, a general disadvantage of a within-subject design is that it is sensitive to practice effects. In our experiment, participants also became better at the task over the course of the three sessions. T2 accuracy increased with session, but this effect was not limited to the attentional blink; it occurred for all lags (cf. Slagter et al. 2007). This general practice effect likely contributed to increased error variance-T2 accuracy differences between sessions that were not due to treatment-decreasing the power to detect an existing effect. However, treatment was counterbalanced across sessions, and we found no effect of treatment order, suggesting that practice did not interact with treatment. Practice effects thus cannot easily account for the null effect of treatment on attentional blink magnitude and, more generally, T2 performance. A second potential limitation of our study is that the majority $(15 / 18)$ of the participants were women and that the menstrual cycle was not taken into account and use of contraceptives was not registered. We are not aware of studies that have reported 
systematic performance differences in RSVP tasks as a function of sex, menstrual cycle, or use of contraceptives (but see Holländer et al. 2005), but we cannot exclude the possibility of such effects in our study.

Acknowledgments This research was supported by a Starting Independent Researcher Grant of the European Research Council (SN) and by the Netherlands Organization for Scientific Research (HAS, SN). The researchers would like to thank Joop van Gerven for his valuable advice about the study design and Marloes van Moort and Annelies de Haan for their assistance in data collection.

Open Access This article is distributed under the terms of the Creative Commons Attribution 4.0 International License (http:// creativecommons.org/licenses/by/4.0/), which permits unrestricted use, distribution, and reproduction in any medium, provided you give appropriate credit to the original author(s) and the source, provide a link to the Creative Commons license, and indicate if changes were made.

\section{References}

Abercrombie ED, Jacobs BL (1987) Single-unit response of noradrenergic neurons in the locus coeruleus of freely moving cats. II. Adaptation to chronically presented stressful stimuli. J Neurosci 7: 2844-2848

Abuljawad KAJ, Langley RW, Bradshaw CM, Szabadi E (2001) Effects of clonidine and diazepam on prepulse inhibition of the acoustic startle response and the N1/P2 auditory evoked potential in man. J Psychopharmacol 15:237-242

Acquas E, Wilson C, Fibiger HC (1998) Pharmacology of sensory stimulation-evoked increases in frontal cortical acetylcholine release. Neuroscience 85:73-83

Adams LM, Foote SL (1988) Effects of locally infused pharmacological agents on spontaneous and sensory-evoked activity of locus coeruleus neurons. Brain Res Bull 21:395-400

Aston-Jones G, Cohen JD (2005) An integrative theory of locus coeruleus-norepinephrine function: adaptive gain and optimal performance. Annu Rev Neurosci 28:403-450

Aston-Jones G, Rajkowski J, Cohen JD (2000) Locus coeruleus and regulation of behavioral flexibility and attention. Prog Brain Res 126:165-182

Beane M, Marrocco RT (2004) Norepinephrine and acetylcholine mediation of the components of reflexive attention: implications for attention deficit disorders. Prog Neurobiol 74:167-181

Bouret S, Sara SJ (2004) Reward expectation, orientation of attention and locus coeruleus-medial frontal cortex interplay during learning. Eur J Neurosci 20:791-802

Briand LA, Gritton H, Howe WM, Young DA, Sarter M (2007) Modulators in concert for cognition: modulator interactions in the prefrontal cortex. Prog Neurobiol 83:69-91

Brown SBRE, Tona KD, van Noorden MS, Giltay EJ, van der Wee NJA, Nieuwenhuis S (2015a) Noradrenergic and cholinergic effects on speed and sensitivity measures of phasic alerting. Behav Neurosci 129:42-49

Brown SBRE, van der Wee NJA, van Noorden MS, Giltay EJ, Nieuwenhuis S (2015b) Noradrenergic and cholinergic modulation of late ERP responses to deviant stimuli. Psychophysiology (in press)

Chun MM, Potter MC (1995) A two-stage model for multiple target detection in rapid serial visual presentation. J Exp Psychol Hum Percept Perform 21:109-127

Colzato LS, Slagter HA, Spapé MM, Hommel B (2008) Blinks of the eye predict blinks of the mind. Neuropsychologia 46:3179-3183
Colzato LS, Slagter HA, de Rover M, Hommel B (2011) Dopamine and the management of attentional resources: genetic markers of striatal D2 dopamine predict individual differences in the attentional blink. J Cogn Neurosci 23:3567-3585

Coull JT (2001) Modulation of attention by noradrenergic $\alpha_{2}$-agents varies according to arousal level. Drug News Perspec 14:5-11

Coull JT, Frith CD, Dolan RJ, Frackowiak RSJ, Grasby PM (1997) The neural correlates of the noradrenergic modulation of human attention, arousal, and learning. Eur J Neurosci 9:589-598

De Martino B, Strange BA, Dolan RJ (2008) Noradrenergic neuromodulation of human attention for emotional and neutral stimuli. Psychopharmacology 197:127-136

dell' Acqua R, Jolicoeur P, Pesciarelli F, Job R, Palomba D (2003) Electrophysiological evidence of visual encoding deficits in a cross-modal attentional blink paradigm. Psychophysiology 40: $629-639$

Dringenberg HC, Vanderwolf CH (1998) Involvement of direct and indirect pathways in electrocorticograph activation. Neurosci Biobehav Rev 22:243-257

Egan TM, North RA (1985) Acetylcholine acts on $\mathrm{m}_{2}$-muscarinic receptors to excite rat locus coeruleus neurones. Br J Pharmacol 85:733735

Gratton G, Coles MGH, Donchin E (1983) A new method for off-line removal of ocular artifact. Electroencephalogr Clin Neurophysiol $55: 468-484$

Hasselmo ME, Sarter M (2011) Modes and models of forebrain cholinergic neuromodulation of cognition. Neuropsychopharmacology 36 : $52-73$

Holländer A, Hausmann M, Hamm JP, Corballis MC (2005) Sex hormonal modulation of hemispheric asymmetries in the attentional blink. J Int Neuropsychol Soc 11:263-272

Hommel B, Akyürek EG (2005) Lag-1 sparing in the attentional blink: benefits and costs of integrating two events into a single episode. Q J Exp Psychol A Hum Exp Psychol 58:1415-1433

Jepma M, Deinum J, Asplund CL, Rombouts SA, Tamsma JT, Tjeerdema N, Spapé MM, Garland EM, Robertson D, Lenders JW, Nieuwenhuis S (2011) Neurocognitive function in dopamine-betahydroxylase deficiency. Neuropsychopharmacology 36:1608-1619

Joseph KC, Sitaram N (1989) The effect of clonidine on auditory P300. Psychiatry Res 28:255-262

Kenemans JL, Kähkönen S (2011) How human electrophysiology informs psychopharmacology: from bottom-up driven processing to top-down control. Neuropsychopharmacology 36:26-51

Key APF, Dove GO, Maguire MJ (2005) Linking brainwaves to the brain: an ERP primer. Dev Neuropsychol 27:183-215

Martens S, Munneke J, Smid H, Johnson A (2006) Quick minds don't blink: electrophysiological correlates of individual differences in attentional selection. J Cogn Neurosci 18:1423-1438

Nieuwenhuis S, Aston-Jones G, Cohen JD (2005a) Decision-making, the $\mathrm{P} 3$, and the locus coeruleus-norepinephrine system. Psychol Bull 131:510-532

Nieuwenhuis S, Gilzenrat MS, Holmes BD, Cohen JD (2005b) The role of the locus coeruleus in mediating the attentional blink: a neurocomputational theory. J Exp Psychol Gen 134:291-307

Nieuwenhuis S, van Nieuwpoort IC, Veltman DJ, Drent ML (2007) Effects of the noradrenergic agonist clonidine on temporal and spatial attention. Psychopharmacology 193:261-269

Pineda JA, Foote SL, Neville HJ (1989) Effects of locus coeruleus lesions on auditory, long-latency, event-related potentials in monkey. $\mathrm{J}$ Neurosci 9:81-93

Raymond JE, Shapiro KL, Arnell KM (1992) Temporary suppression of visual processing in an RSVP task: an attentional blink? J Exp Psychol Hum Percept Perform 18:849-860

Rolke B, Heil M, Streb J, Hennighausen E (2001) Missed prime words within the attentional blink evoke an N400 semantic priming effect. Psychophysiology 38:165-174 
Sergent C, Baillet S, DeHaene S (2005) Timing of the brain events underlying access to consciousness during the attentional blink. Nat Neurosci 8:1391-1400

Servan-Schreiber D, Prinz H, Cohen JD (1990) A network model of catecholamine effects: gain, signal-to-noise ratio, and behavior. Science 249:892-895

Slagter HA, Lutz A, Greischar LL, Francis AD, Nieuwenhuis S, Davis JM, Davidson RJ (2007) PLoS Biol 5:e138

Slagter HA, Tomer R, Christian BT, Fox AS, Colzato LS, King CA, Murali D, Davidson RJ (2012) PET evidence for a role for striatal dopamine in the attentional blink: functional implications. J Cogn Neurosci 24:1932-1940

Śmigasiewicz K, Weinrich J, Reinhardt B, Verleger R (2014) Deployment and release of interhemispheric inhibition in dual-stream rapid serial visual presentation. Biol Psychol 99:47-59
Smith A, Nutt D (1996) Noradrenaline and attention lapses. Nature 380:291

Stewart C, Burke S, Marrocco R (2001) Cholinergic modulation of covert attention in the rat. Psychopharmacology 155:210-218

Turetsky BI, Fein G (2002) $\alpha 2$-Noradrenergic effects on ERP and behavioral indices of auditory information processing. Psychophysiology 39:147-157

Usher MU, Cohen JD, Servan-Schreiber D, Rajkowski J, Aston-Jones G (1999) The role of locus coerulues in the regulation of cognitive performance. Science 283:549-554

Warren CM, Breuer AT, Kantner J, Fiset D, Blais C, Masson ME (2009) Target-distractor interference in the attentional blink implicates the locus coeruleus-norepinephrine system. Psychon Bull Rev 16:11061111 